\title{
Functional Associations between Gut Microbiome and Bone Health Status in Post-Menopausal Women: A Cross-Sectional Study ${ }^{\dagger}$
}

\author{
Bolaji Lilian Ilesanmi-Oyelere ${ }^{1,2,3, *}$, Elizabeth Rettedal ${ }^{2,3}$, Nicole C. Roy ${ }^{2,3,4}$, Jane Coad ${ }^{5}$ and \\ Marlena C. Kruger 1,3 \\ 1 School of Health Sciences, College of Health, Massey University, Tennent Drive, Palmerston North 4442, \\ New Zealand; m.c.kruger@massey.ac.nz \\ 2 Food Nutrition \& Health Team, AgResearch Grasslands, Palmerston North 4442, New Zealand; \\ elizabeth.rettedal@agresearch.co.nz (E.R.); nicole.roy@agresearch.co.nz (N.C.R.) \\ 3 Riddet Institute, Massey University, Palmerston North 4442, New Zealand \\ 4 High-Value Nutrition National Science Challenge, Auckland 1142, New Zealand; \\ 5 School of Food and Advanced Technology, College of Sciences, Massey University, Tennent Drive, \\ Palmerston North 4442, New Zealand; j.coad@massey.ac.nz \\ * Correspondence: b.ilesanmi-oyelere@massey.ac.nz; Tel.: +64-(0)21-0852-2308 \\ + Presented at the 2019 Annual Meeting of the Nutrition Society of New Zealand, Napier, New Zealand, \\ 28-29 November 2019.
}

Published: 16 December 2019

Introduction: The gut microbiome, the totality of micro-organisms and their collective genetic makeup plays a significant role in bone health. Similarly, pro-inflammatory cytokines influence bone health status. The gut microbiome has been postulated to affect nutrient absorption, endocrine and immune systems important in bone metabolism. Our aim was to determine the functional relationship between gut microbiome and bone health status in postmenopausal women. Methods: The study involved 86 eligible post-menopausal women. Dual energy X-ray Absorptiometry (DXA) was used to measure body composition and femoral neck (FN), lumbar spine (LS), and total hip bone mineral density (BMD). Faecal DNA samples were collected and underwent library preparation by Nextera XT DNA library prep kit (Illumina, San Diego, CA, USA) and shotgun metagenomic sequencing was conducted on an Illumina HiSeq ${ }^{\circledR} 2500$ System. Shotgun metagenome data were processed in MG-RAST and analysed/visualised in MicrobiomeAnalyst. The KEGG Orthology (KO) database was used. Results: Functional metagenome of the gut microbiota were significantly different between the healthy and osteoporotic women, defined by both the hip and femoral neck T-scores, based on the Interferon gamma receptor 1 (CD119) pathway (FDR $P$-value $<0.05$ ). There were also significant differences in the fructuronate reductase (uxuB), nucleoside-diphosphate kinase, DNAdirected RNA polymerase II subunit (RPB11) and DNA-directed RNA polymerase subunit omega (rpoZ) pathways (FDR $P$-value $<0.05$ ) based on the femoral neck T-score classification. However, we found no differences in the molecular function between the healthy and osteoporotic women based on the spine T-scores. Conclusion: The inflammatory osteoclastogenic cytokine, interferon gamma seems to be an important determining cytokine for bone loss. It is known to contribute to osteoclast differentiation by enhancing the signalling pathways. Our results provide a basis for a longitudinal intervention to assess the role of the gut microbiome composition and function in bone health for preventive and therapeutic interventions in postmenopausal women.

(C) 2019 by the authors. Licensee MDPI, Basel, Switzerland. This article is an open access article distributed under the terms and conditions of the Creative Commons Attribution (CC BY) license (http://creativecommons.org/licenses/by/4.0/). 